\title{
A Soft Rough-Fuzzy Preference Set-Based Evaluation Method for High-Speed Train Operation Diagrams
}

\author{
Dingjun Chen, ${ }^{1,2,3}$ Shaoquan Ni, ${ }^{1,2,3}$ Chang'an Xu, ${ }^{1,2,3}$ Hongxia Lv, ${ }^{1,2,3}$ and Keyun Qin ${ }^{4}$ \\ ${ }^{1}$ School of Transportation and Logistics, Southwest Jiaotong University, Chengdu 610031, China \\ ${ }^{2}$ National Railway Train Diagram Research and Training Center, Southwest Jiaotong University, Chengdu 610031, China \\ ${ }^{3}$ National and Local Joint Engineering Laboratory of Comprehensive Intelligent Transportation, Chengdu 610031, China \\ ${ }^{4}$ School of Mathematics, Southwest Jiaotong University, Chengdu 610031, China
}

Correspondence should be addressed to Shaoquan Ni; shaoquanni@163.com

Received 11 November 2015; Accepted 31 January 2016

Academic Editor: Mingshu Peng

Copyright (C) 2016 Dingjun Chen et al. This is an open access article distributed under the Creative Commons Attribution License, which permits unrestricted use, distribution, and reproduction in any medium, provided the original work is properly cited.

\begin{abstract}
This paper proposes a method of high-speed railway train operation diagram evaluation based on preferences of locomotive operation, track maintenance, S \& C, vehicles and other railway departments, and customer preferences. The application of rough set-based attribute reduction obtains the important relative indicators by eliminating excessive and redundant evaluation indicators. Soft fuzzy set theory is introduced for the overall evaluation of train operation diagrams. Each expert utilizes a set of indicators during evaluation based on personal preference. In addition, soft fuzzy set theory is applied to integrate the information obtained via expert evaluation in order to obtain an overall evaluation. The proposed method was validated by a case study. Results demonstrate that the proposed method flexibly expresses the subjective judgments of experts while effectively and reasonably handling the uncertainty of information, which is consistent with the judgment process of humans. The proposed method is also applicable to the evaluation of train operation schemes which consist of multiple diagrams.
\end{abstract}

\section{Introduction}

The transportation and production activities of railways are integrated into train operation diagrams which involve train operation, locomotive operation, track maintenance, S \& C, vehicles, and other departments. This train operation diagram is the basis of overall planning and train operation organization in railway transportation, as well as the primary tool for coordination among various departments within the railway network and maintaining a working order of production activities. Train operation diagrams are compiled according to the requirements of national economic development and railway transportation capacity. The process reflects various qualitative and quantitative indicators of railway operation. The compilation quality of a train operation diagram directly affects the safety and benefits of railway transportation, as well as the adaptability of the railway system to social and economic development. The transportation organization ability of the railway system is reflected by the quality of high-speed railway train operation diagrams, the evaluation of which guides potential improvements to train operation diagrams. Therefore, the evaluation of train operation diagrams is of great importance in order to maintain operation safety, adapt to market demands, and improve transportation capacity, efficiency, and benefits.

Extensive studies have been conducted to evaluate highspeed railway train operation diagrams, and many valuable achievements have been reported. In foreign countries, train operation diagrams are often cyclic with standardized structures; thus the related studies are relatively simple. Most such studies have focused on the propagation and impacts of train delays, which primarily involve indicators such as the reliability and stability of diagrams. Carey $[1,2]$ utilized probability theory to describe the propagation of train delays and proposed a method to configure buffer time. Hansen [3] identified associative delay as an indicator for the initial evaluation of train operation diagrams. Goverde $[4,5]$ and Delorme et al. [6] further refined the identified indicators. 
Börjesson and Eliasson [7] and Vromans et al. [8] proposed the use of the average delay time and heterogeneity as indicators to evaluate train operation diagrams. Goverde and Yan [9] defined delay impact, delay sensitivity, and delay propagation as indicators to analyze timetable stability and robustness. In China, however, noncyclic train operation diagrams are typical, which usually incorporate complex structures without universal standards. Related studies have focused primarily on analysis of the technical standards for train operation diagrams, including evaluation indicators for basic operation diagrams, quality of passenger service, balance, adjustability, and adaptability. Yang and $\mathrm{Hu}$ [10] first proposed a dynamic train operation diagram indicator index to evaluate Chinese railway systems. Tian [11] proposed the division of train operation diagram evaluation indicators into two categories: static indicators and dynamic indicators. Peng $[12,13]$ proposed the assessment of diagram balance via deviation and bias and the description of diagram adjustability via the recovery of train delay. Meng and Jia [14] evaluated the quality of the timetable from the interior and exterior factors affecting the timetable stability. Liu [15] proposed using the train timetable structure and delay propagation in a railway network as indices to evaluate the timetable robustness. Ma et al. [16], Zhu et al. [17], and Bao [18] constructed relatively complete train operation diagram evaluation indices from various perspectives.

Max-plus theory and random simulation are common methods for the evaluation of train operation diagrams based on patterns of delay propagation and exploit the cyclic characteristic of operation diagrams by utilizing the lines within a cycle as a study unit. Carey [1,2], Sipilä [19], and Keiji et al. [20] performed a systematic study of train delay propagation patterns via random simulation. Hansen [3], Goverde [4, 5], and Vromans et al. [8] applied max-plus theory to the stability evaluation of cyclic train operation diagrams. Higgins and Kozan [21] and Salido et al. [22] evaluated the stability of train operation diagrams via experimental simulations. In China, due to the structural complexity of train operation diagrams, scholars have attempted fewer analyses regarding the impacts of train delays. Most recent studies have focused on evaluation of technical indicators of train operation diagrams and interactions among indicators by applying mathematical methods such as fuzzy synthetic evaluation, grey theory, and random simulation. Tian [11] and Zhu et al. [17] applied the fuzzy synthetic method to evaluate the quality of train operation diagrams. Peng $[12,13]$ and Bao [18] evaluated train operation diagrams via fuzzy decisionmaking matrix theory and grey theory, respectively. Yang and $\mathrm{He}$ [23] and Meng et al. [24] adopted random simulation and max-plus theory to evaluate Chinese train operation diagrams. Jiang et al. [25] presented a model based on timedriven microscopic simulation to evaluate train timetable from the viewpoint of big passengers' data on rail transit lines. Meng and Jia [14] defined the information entropy of the exterior factors and the interior factors affecting the timetable stability to evaluate the quality of the train timetable.

Due to significant differences in railway transportation histories, diagram compilation systems, and diagram structures, studies of train operation diagrams in China differ significantly from methods used abroad, particularly in regard to indicator construction and method selection. Most studies of train operation diagrams worldwide have focused on the performance of technical indicators and relationships among indicators. In particular, interference propagation and stability evaluations of cyclic operation diagrams have been extensively studied.

Multiple train operation diagrams for further selection can be made in a short time with the aid of computers. The evaluation of train operation diagrams requires the identification of an optimal diagram from multiple options via the coordination of multiple experts. Each diagram represents a comprehensive plan for railway transportation including train operation, locomotive operation, track maintenance, $S$ $\& \mathrm{C}$, vehicles, and other departments involved in a railway system. Thus, a committee is responsible for the compilation of train operation diagrams, and a workgroup is formed consisting of representatives from the following departments: the office of the chief engineer, transportation management, passenger traffic, power supply, vehicles, track maintenance, $S \& C$, the dispatching station, and joint-venture railway companies. The workgroup responds to technical problems during diagram compilation, organizes investigations and traction tests, inspects technical work and time standards, verifies and reports compilation data, and is responsible for the compilation, adjustment, and implementation of the train operation diagram. However, each of the many departments of a railway system and the customers participating in the compilation and evaluation possess different knowledge, experiences, and perspectives and thus may only focus on the indicators representative of their own interests and/or familiarity. If all evaluators were required to evaluate all possible indicators, large differences may appear in evaluation results, resulting in potential misjudgments in the final decision making. Therefore, this study constructed an evaluation index for high-speed train operation diagrams based on the preferences of different railway departments and customers. Redundant indicators were reduced by applying the rough set-based attribute reduction method to identify the relatively important indicators. Soft fuzzy set theory was employed in order to comprehensively evaluate the quality of operation diagrams in consideration of the different preferences among experts.

\section{Preference-Based High-Speed Train Operation Diagram Evaluation Indices}

The compilation of train operation diagrams is a complex task which requires collaboration across various departments. In this study, existing studies of train operation diagram evaluation were summarized, and a high-speed train operation diagram evaluation index was established based on the preferences of the transportation department, passenger traffic department, vehicles department, railway stations, and passengers.

2.1. High-Speed Train Operation Diagram Evaluation Indicators Based on Preferences of the Transportation Department. The railway transportation department is the primary 
compilation agent for train operation diagrams and is ultimately responsible for the compilation and management of the diagrams. A panel of personnel is assigned to execute the technical work standards for compilation and is responsible for the compilation of train operation diagrams, database maintenance, implementation of and adjustments to the diagrams, and analysis of operation capacity and diagram indicators. Indicators include the number of local-line trains, cross-line trains, technical speed, travel speed, speed coefficient, electric multiple unit (EMU) usage, EMU turn-around time, EMU connection time, EMU daily mileage, EMU utilization balance, EMU utilization rate, stop time, number of stops, average stop time, operation diagram balance, and capability utilization.

2.2. High-Speed Train Operation Diagram Evaluation Indicators Based on Preferences of the Passenger Traffic Department. The primary duties of the passenger traffic department in the compilation of train operation diagrams include proposing line plans, providing product requirements and related data, and performing product quality calculations and analysis. During compilation, they also verify the related working and timing standards. Their preferred indicators include train service frequency, peak hour departure frequency, reasonability coefficient of departure and arrival times, the average number of stops, average stop distance, travel speed, stop time, total number of stops, average stop time, service station stop time, number of service station stops, nonservice station stop time, number of nonservice station stops, stop plan balance, profile passenger flow adaptability, travel time loss, average number of transfers, accessibility coefficient, average cross-line through speed, economic benefit, social benefit, market benefit, average train buffer time, number of backup lines, diagram balance, number of delayed trains, delay time, probability of delay, delay recovery, and buffer time utilization.

\subsection{High-Speed Train Operation Diagram Evaluation Indi-} cators Based on Preferences of the Vehicles Department. The vehicles department of the railway administration primarily provides data, determines the deployment of EMUs, plans maintenance, and formulates crew schedules and the EMU routing scheme. Additionally, they review the technical work and time standards during diagram compilation. Their preferred indicators include EMU routing mileage, routing time, number of drivers, number of crew members, EMU usage, EMU turn-around time, EMU connection time, EMU daily mileage, EMU utilization balance, EMU utilization rate, average EMU mileage, total mileage, crew cost, working time, and work intensity balance.

2.4. High-Speed Train Operation Diagram Evaluation Indicators Based on Preferences of Railway Stations. Train stations allow for safe travel and quick distribution of passengers, ensuring that passengers can follow travel procedures with speed and ease, and provide comfortable passenger waiting environments as well as beneficial cultural and life services. Train stations also accomplish the carrying, handling, holding, and delivery of luggage and packages. Train operation diagrams and the technical work of train stations both influence and constrain one another. High-quality train operation diagrams influence the smoothness of work at train stations, whereas the capacities of train stations constrain the implementation of the train operation diagram. The preferred indicators of train stations include the total number of train pairs, the number of from-station pairs, the number of tostation pairs, cost performance, the number of train pairs at service transfer stations, the number of train pairs at nonservice transfer stations, train service frequency, degree of train delay, and train operation diagram balance.

2.5. High-Speed Train Operation Diagram Evaluation Indicators Based on Preferences of Passengers. Passengers have developed increasingly higher demands for quality of travel with economic development and the parallel improvement of general living standards. The departure times of intercity and high-speed trains must be regular and cyclic in order to serve fluctuating passenger traffic at various times. For passenger traffic that cannot be processed by direct traffic, the interchange must be carefully designed so as to result in a reasonable transfer time. The preferred indicators of passengers include reasonability of departure time distribution, direct traffic index, accessibility index, wait time satisfaction, loss of travel time, travel speed, average number of transfers, cost performance rating, train service frequency, and the possibility of delay.

\section{Soft Rough-Fuzzy Preference Set-Based Evaluation of High-Speed Train Operation Diagrams}

The proposed high-speed train operation diagram evaluation index consists of a large number of indicators, many of which are redundant. Additionally, expert evaluation is reasonably subjective. In this study, rough set-based attribute reduction was first applied to eliminate redundant indicators. Next, the different preferences of various experts were processed by applying soft fuzzy set theory. Results of evaluations conducted by different experts were then integrated in order to obtain an overall evaluation.

3.1. Rough Set Theory. Fuzzy set theory was first introduced by Polish mathematician Pawlak in 1982, and it offers unique advantages in the handling of incomplete and low-accuracy data. The application of fuzzy set theory can eliminate redundant information in data sets while maintaining the inherent classification ability of the data $[26,27]$.

Definition 1. The two-element set $(U, \mathbb{R})$ is a knowledge base in which $U$ is the domain, and $\mathbb{R}$ is a family of equivalence relations on $U$. If $P \subseteq \mathbb{R}$, then $\bigcap P=\operatorname{ind}(P)$ is another equivalent relation, known as an indiscernibility relation on $P$.

Definition 2. Let $\mathbb{R}$ be a family of equivalence relations, and $R \in \mathbb{R}$. If ind $(\mathbb{R})=\operatorname{ind}(\mathbb{R}-\{R\})$, then $R$ is unnecessary in $\mathbb{R}$; if not, it is necessary in $\mathbb{R}$. If every $R \in \mathbb{R}$ is necessary in $\mathbb{R}$, then $\mathbb{R}$ is classified as independent. For $Q \subseteq P$, if $Q$ is independent and $\operatorname{ind}(Q)=\operatorname{ind}(P)$, then $Q$ is a reduction of $P$. 
3.2. Soft Fuzzy Preference Set. Soft set theory was first proposed by Molodtsov in 1999, and is commonly used as a mathematical tool for analysis of uncertain objects, fuzzy objects, or objects that cannot be precisely defined. Soft set theory is often applied to decision-making, evaluation, attribute reduction, soft algebra, prediction, and classification $[28,29]$.

Definition 3 (soft set). Let $U$ represent the initial domain, $E$ the parameter set, and $P(U)$ the power set of $U .(F, E)$ is a soft set on $U$ if and only if $F$ is a mapping of $E$ to $P(U)$.

$\forall e \in E, F(e)$ is the set of elements in $U$ that exhibit the properties of attribute $e$; that is, $F(e) \subseteq U$. Meanwhile, soft set $(F, E)$ is an approximate set composed of multiple sets, each of which exhibits the properties of the elements in $U$.

Definition 4 (soft fuzzy set). Let $U$ represent the initial domain, $E$ the parameter set, $\xi(U)$ a fuzzy set defined on $U$, and $A \subseteq E$. Therefore, $(F, A)$ is a soft fuzzy set on domain $U$ if and only if $F$ is a mapping of $A$ to $\xi(U)$.

Definition 5 (“AND” operation). Let $(F, A)$ and $(G, B)$ represent two soft fuzzy sets on $U$. If, $\forall(\alpha, \beta) \in(A, B), H(\alpha, \beta)=$ $F(\alpha) \cap G(\beta)$, then $(F, A) \wedge(G, B)=(H, A \times B)$ represents the “AND" operation of $(F, A)$ and $(G, B)$.

3.3. Representation of Uncertain Information in the Evaluation of High-Speed Train Operation Diagrams. Let YXT = $\left\{y x t_{1}, y x t_{2}, \ldots, y x t_{n}\right\}$ denote a set of train operation diagrams to be evaluated, where $y x t_{i}(i=1,2, \ldots, n)$ denotes the $i$ th operation diagram, $C=\left\{c_{1}, c_{2}, \ldots, c_{m}\right\}$ is the set of evaluation indicators, and $\mathrm{EX}=\left\{\mathrm{ex}_{1}, \mathrm{ex}_{2}, \ldots, \mathrm{ex}_{p}\right\}$ represents the set of experts. Considering the differences in knowledge, experience, and perspectives of the various experts, the evaluation indicator set of expert $\operatorname{ex}_{k}(k=1,2, \ldots, p)$ is denoted by $C_{k}=\left\{c_{1}^{k}, c_{2}^{k}, \ldots, c_{l_{k}}^{k}\right\}$, where $c_{j}^{k} \in C(j=$ $\left.1,2, \ldots, l_{k}\right)$. The evaluation matrix of expert $\operatorname{ex}_{k}$ on evaluation indicator set $C_{k}$ for the operation diagram set $Y X T$ is $V_{k}=$ $\left(v_{i j}^{k}\right)_{n \times l_{k}}$, where $v_{i j}^{k}$ denotes the value assigned by the expert to indicator $c_{j}^{k}$ for diagram $y x t_{i}$.

Certain indicators are difficult to describe qualitatively or quantitatively, such as social benefit and wait time satisfaction. Simple affirmation or negation cannot accurately express expert opinions, while phrases such as "very good," "good," and "fair" are useful, but ambiguous. In this study, the expert evaluations of high-speed train operation diagrams are scaled as $H=\{0,0.1,0.2,0.3,0.4,0.5,0.6,0.7,0.8,0.9,1.0\}$.

\subsection{Evaluation Procedure and Algorithm}

3.4.1. Indicator Reduction. For the evaluation index $C$ of high-speed train operation diagrams, the reduced indicator set is calculated according to the formula ind $(\mathbb{R})=\operatorname{ind}(\mathbb{R}-$ $\{R\})$. For convenience, the reduced evaluation index is still denoted by $C$.

3.4.2. Description of Evaluation Matrix with Soft Fuzzy Set. Based on the evaluation indicator set $C_{k}$ and the evaluation

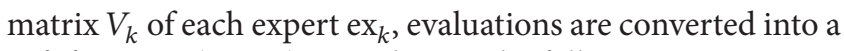
soft fuzzy set $\left(F_{k}, C_{k}\right)$ according to the following equations:

$$
\begin{aligned}
\left(F_{k}, C_{k}\right)=\left\{c_{1}^{k}=\left\{\frac{y x t_{1}}{v_{11}^{k}}, \frac{y x t_{2}}{v_{21}^{k}}, \ldots, \frac{y x t_{n}}{v_{n 1}^{k}}\right\}, c_{2}^{k}\right. \\
\quad=\left\{\frac{y x t_{1}}{v_{12}^{k}}, \frac{y x t_{2}}{v_{22}^{k}}, \ldots, \frac{y x t_{n}}{v_{n 2}^{k}}\right\}, \ldots, c_{2}^{k} \\
=\left\{\frac{y x t_{1}}{v_{12}^{k}}, \frac{y x t_{2}}{v_{22}^{k}}, \ldots, \frac{y x t_{n}}{v_{n 2}^{k}}\right\}, \ldots, c_{l_{k}}^{k} \\
\left.=\left\{\frac{y x t_{1}}{v_{1 l_{k}}^{k}}, \frac{y x t_{2}}{v_{2 l_{k}}^{k}}, \ldots, \frac{y x t_{n}}{v_{n l_{k}}^{k}}\right\}\right\} .
\end{aligned}
$$

3.4.3. Information Integration. Through “AND” operations, the evaluations of each individual expert are integrated to obtain an overall evaluation matrix. "AND" operations are applied to sets $\left(F_{1}, E_{1}\right),\left(F_{2}, E_{2}\right), \ldots$, and $\left(F_{m}, E_{m}\right)$; the calculation result is denoted as $(G, E)$, and

$$
\begin{aligned}
(G, E) & =\left(G, C_{1} \times C_{2} \times \cdots \times C_{m}\right) \\
& =\left(F_{1}, C_{1}\right) \wedge\left(F_{2}, C_{2}\right) \wedge \cdots \wedge\left(F_{m}, C_{m}\right) .
\end{aligned}
$$

$(G, E)$ is also a soft fuzzy set, with parameters composed of the evaluation indicator sets $C_{1}, C_{2}, \ldots$, and $C_{m}$ of the $m$ experts. Assuming that $(G, E)$ consists of $L$ parameters, let $E=\left\{e_{1}, e_{2}, \ldots, e_{L}\right\}$. Thus, $(G, E)$ can be expressed as follows:

$$
\begin{aligned}
& (G, E)=\left\{e_{1}=\left\{\frac{s_{1}}{\mu_{11}}, \frac{s_{2}}{\mu_{21}}, \ldots, \frac{s_{q}}{\mu_{q 1}}\right\}, e_{2}=\left\{\frac{s_{1}}{\mu_{12}},\right.\right. \\
& \left.\left.\frac{s_{2}}{\mu_{22}}, \ldots, \frac{s_{q}}{\mu_{q 2}}\right\}, \ldots, e_{L}=\left\{\frac{s_{1}}{\mu_{1 L}}, \frac{s_{2}}{\mu_{2 L}}, \ldots, \frac{s_{q}}{\mu_{q L}}\right\}\right\} \text {. } \\
& \text { If } C_{1} \cap C_{2} \cap \cdots \cap C_{m}=\varnothing \text {, then } \mu_{i j}= \\
& \min _{\tilde{c}_{x}^{j} \in\left\{\tilde{c}_{1}^{j}, \tilde{c}_{2}^{j}, \ldots, \tilde{c}_{m}^{j}\right\}, k \in\{1,2, \ldots, m\}}\left\{v_{t x}^{k}\right\} \text {. } \\
& \text { If } C_{1} \cap C_{2} \cap \cdots \cap C_{m} \neq \varnothing \text {, then } \\
& \mu_{i j}=\min \left\{\min _{\widetilde{c}_{x}^{j} \in\left\{\tilde{c}_{1}^{j}, \tilde{c}_{2}^{j}, \ldots, \widetilde{c}_{m}^{j}\right\}, k \in\{1,2, \ldots, m\}}\left\{v_{t x}^{k}\right\}, \lambda_{t}\right\} \text {, } \\
& \lambda_{t}=\text { average }_{k \in\left\{k_{1}, k_{2}, \ldots, k_{m}\right\}}\left\{v_{t p}^{k}\right\} \text {, }
\end{aligned}
$$

where $\mu_{i j}$ indicates the degree to which the evaluated operation scheme $s_{t}$ matches the synthesized parameter $e_{j}(j=$ $1,2, \ldots, L)$.

3.4.4. Overall Evaluation. The comparison table CT= $\left(\mathrm{ct}_{x y}\right)_{q \times q}$ is computed, in which $\mathrm{ct}_{x y}=\sum_{j} \gamma_{x y}^{j}$,

$$
\gamma_{x y}^{j}= \begin{cases}1, & u_{x j} \geq u_{y j}, \\ 0, & u_{x j}<u_{y j}\end{cases}
$$

and $\mathrm{ct}_{x y}$ indicates the number of evaluation indicators that are assigned higher values for diagram $y x t_{x}$ than those assigned to evaluation indicators for diagram $y x t_{y}$. 


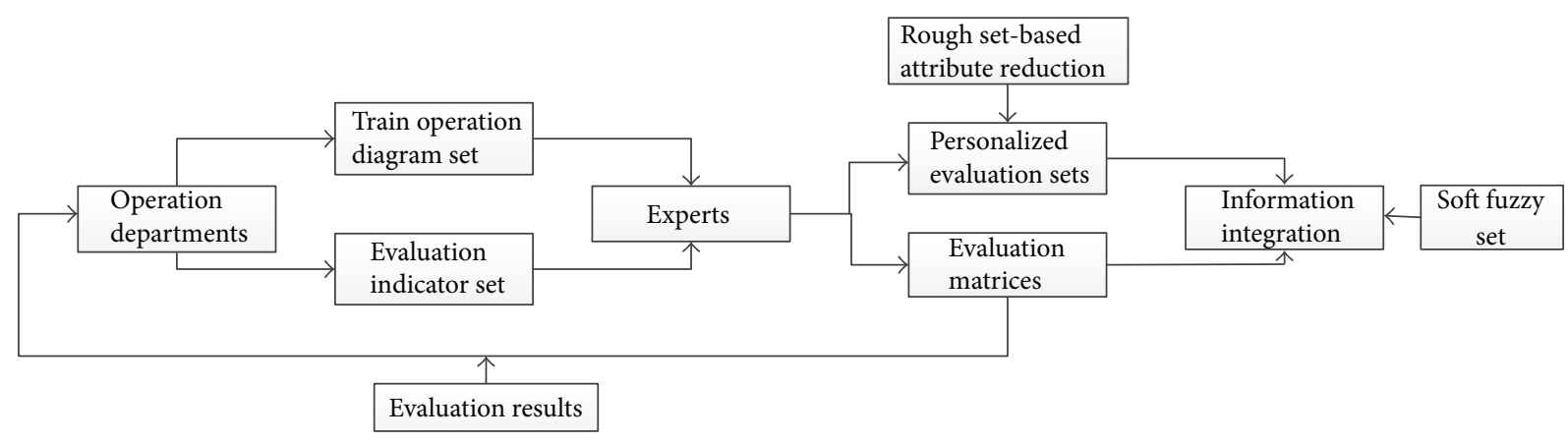

FigurE 1: Flowchart of the soft fuzzy preference-based evaluation of high-speed train operation diagrams.

According to the comparison table CT, the evaluated score score $\left(y x t_{t}\right)$ for each train operation diagram can be calculated by $\operatorname{score}\left(y x t_{t}\right)=r_{x}-t_{x}$, where $r_{x}=\sum_{y=1}^{q} \mathrm{ct}_{x y}$ and $t_{y}=\sum_{x=1}^{q} \mathrm{ct}_{x y}$.

In the above equation, $r_{x}$ represents the number of indicators that were assigned higher evaluations for diagram $y x t_{x}$ than other members of YXT, $t_{y}$ represents the number of indicators that were assigned higher evaluations for diagram $y x t_{y}$ than other members of YXT, and score $\left(y x t_{t}\right)$ describes the superiority of $y x t_{t}$ in YXT. Larger values of score $\left(y x t_{t}\right)$ indicate that $y x t_{t}$ is more optimal.

\subsubsection{Algorithm}

Step 1. Form the high-speed train operation diagram evaluation index $A$.

Step 2. Reduce the indicators of $A$ by applying rough setbased attribute reduction, and obtain the reduced indicator set $A^{\prime}$.

Step 3. Apply soft rough set theory to integrate the evaluations from all experts, and obtain a final evaluation.

\subsubsection{Flow Diagram of the Algorithm. See Figure 1.}

\section{Case Study}

Two-train operation diagrams shown in Figures 2 and 3 were compiled according to different decision-making approaches, based on actual data reported by the BeijingShanghai high-speed railway system. The two diagrams are denoted by $Y X T=\left\{y x t_{1}, y x t_{2}\right\}$. Administrative staffs from the following departments were selected as experts: transportation, passenger traffic, vehicles, and stations. Based on their individual preferences, an evaluation index $C$ was established and then reduced via rough set-based attribute reduction. Indicators of the index include operation diagram balance $\left(c_{1}\right)$, travel speed $\left(c_{2}\right)$, EMU turn-around time $\left(c_{3}\right)$, train frequency $\left(c_{4}\right)$, cost performance $\left(c_{5}\right)$, and possibility of delay $\left(c_{6}\right)$. The evaluation index is denoted by $C=\left\{c_{1}, c_{2}, \ldots, c_{6}\right\}$. Four experts, $\mathrm{EX}=\left\{\mathrm{ex}_{1}, \mathrm{ex}_{2}, \mathrm{ex}_{3}, \mathrm{ex}_{4}\right\}$, evaluated the compiled train operation diagrams based on the established evaluation index.
Based on their knowledge and experience, each expert provided personal evaluation sets $C_{1}=\left\{c_{1}, c_{2}, c_{3}\right\}, C_{2}=$ $\left\{c_{1}, c_{2}, c_{4}, c_{6}\right\}, C_{3}=\left\{c_{3}\right\}$, and $C_{4}=\left\{c_{1}, c_{4}, c_{5}\right\}$, as well as corresponding evaluation matrices $V_{1}, V_{2}, V_{3}$, and $V_{4}$, where

$$
\begin{aligned}
& c_{1}, \quad c_{2}, \quad c_{3} \\
& V_{1}=\begin{array}{l}
y x t_{1} \\
y x t_{2}
\end{array}\left(\begin{array}{lll}
0.4 & 0.7 & 0.6 \\
0.4 & 0.6 & 0.5
\end{array}\right), \\
& c_{1}, \quad c_{2}, \quad c_{4}, \quad c_{6} \\
& V_{2}=\begin{array}{l}
y x t_{1} \\
y x t_{2}
\end{array}\left(\begin{array}{llll}
0.6 & 0.6 & 0.7 & 0.8 \\
0.5 & 0.7 & 0.7 & 0.6
\end{array}\right), \\
& c_{3} \\
& V_{3}=\begin{array}{ll}
y x t_{1} \\
y x t_{2}
\end{array}\left(\begin{array}{l}
0.7 \\
0.6
\end{array}\right), \\
& c_{1}, \quad c_{4}, \quad c_{5} \\
& V_{4}={ }_{y x t_{2}}^{y x t_{1}}\left(\begin{array}{lll}
0.6 & 0.7 & 0.7 \\
0.5 & 0.6 & 0.8
\end{array}\right) .
\end{aligned}
$$

Then,

$$
\begin{aligned}
& \left(F_{1}, C_{1}\right)=\left\{c_{1}=\left\{\frac{y x t_{1}}{0.4}, \frac{y x t_{2}}{0.4}\right\}, c_{2}\right. \\
& \left.=\left\{\frac{y x t_{1}}{0.7}, \frac{y x t_{2}}{0.6}\right\}, c_{3}=\left\{\frac{y x t_{1}}{0.6}, \frac{y x t_{2}}{0.5}\right\}\right\}, \\
& \left(F_{2}, C_{2}\right)=\left\{c_{1}=\left\{\frac{y x t_{1}}{0.6}, \frac{y x t_{2}}{0.5}\right\}, c_{2}\right. \\
& =\left\{\frac{y x t_{1}}{0.6}, \frac{y x t_{2}}{0.7}\right\}, c_{4}=\left\{\frac{y x t_{1}}{0.7}, \frac{y x t_{2}}{0.7}\right\}, c_{6} \\
& \left.=\left\{\frac{y x t_{1}}{0.8}, \frac{y x t_{2}}{0.6}\right\}\right\}, \\
& \left(F_{3}, C_{3}\right)=\left\{c_{3}=\left\{\frac{y x t_{1}}{0.7}, \frac{y x t_{2}}{0.6}\right\}\right\}, \\
& \left(F_{4}, C_{4}\right)=\left\{c_{1}=\left\{\frac{y x t_{1}}{0.6}, \frac{y x t_{2}}{0.5}\right\}, c_{4}\right. \\
& \left.=\left\{\frac{y x t_{1}}{0.7}, \frac{y x t_{2}}{0.6}\right\}, c_{5}=\left\{\frac{y x t_{1}}{0.7}, \frac{y x t_{2}}{0.8}\right\}\right\} .
\end{aligned}
$$




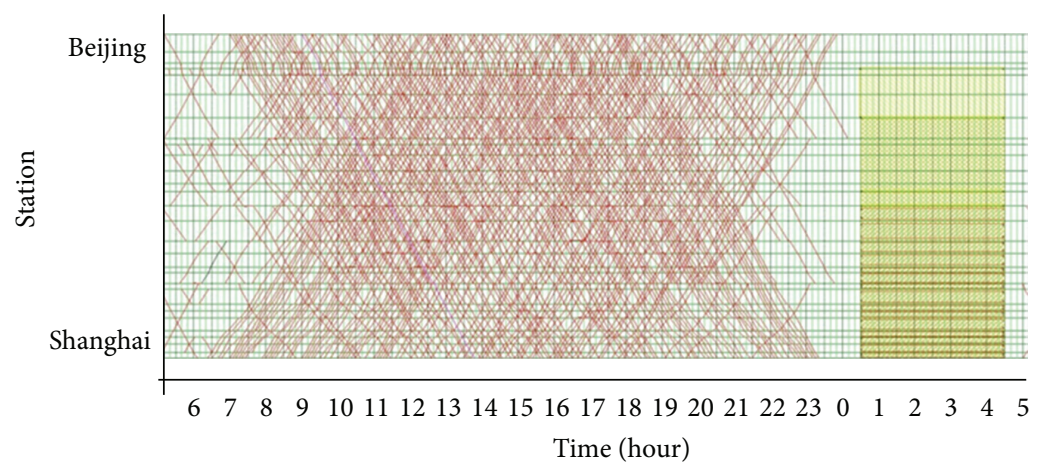

FIGURE 2: Beijing-Shanghai high-speed railway train operation diagram $\left(y x t_{1}\right)$.

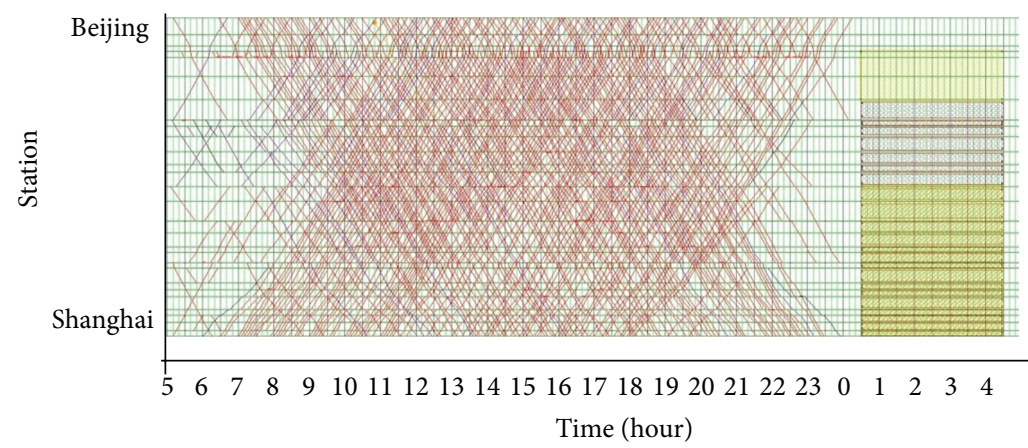

FIGURE 3: Beijing-Shanghai high-speed railway train operation diagram $\left(y x t_{2}\right)$.

TABLE 1: Parameters of $E$.

\begin{tabular}{lcccccc}
\hline$e_{j}$ & $e_{1}$ & $e_{2}$ & $e_{3}$ & $e_{4}$ & $e_{5}$ & $e_{6}$ \\
\hline Parameters & $c_{1} c_{1} c_{3} c_{1}$ & $c_{1} c_{1} c_{3} c_{4}$ & $c_{1} c_{1} c_{3} c_{5}$ & $c_{1} c_{2} c_{3} c_{1}$ & $c_{1} c_{2} c_{3} c_{4}$ & $c_{1} c_{2} c_{3} c_{5}$ \\
\hline$e_{j}$ & $e_{7}$ & $e_{8}$ & $e_{9}$ & $e_{10}$ & $e_{11}$ & $e_{12}$ \\
\hline Parameters & $c_{1} c_{4} c_{3} c_{1}$ & $c_{1} c_{4} c_{3} c_{4}$ & $c_{1} c_{4} c_{3} c_{5}$ & $c_{1} c_{6} c_{3} c_{1}$ & $c_{1} c_{6} c_{3} c_{4}$ & $c_{1} c_{6} c_{3} c_{5}$ \\
\hline$e_{j}$ & $e_{13}$ & $e_{14}$ & $e_{15}$ & $e_{16}$ & $e_{17}$ & $e_{18}$ \\
\hline Parameters & $c_{2} c_{1} c_{3} c_{1}$ & $c_{2} c_{1} c_{3} c_{4}$ & $c_{2} c_{1} c_{3} c_{5}$ & $c_{2} c_{2} c_{3} c_{1}$ & $c_{2} c_{2} c_{3} c_{4}$ & $c_{2} c_{2} c_{3} c_{5}$ \\
\hline$e_{j}$ & $e_{19}$ & $e_{20}$ & $e_{21}$ & $e_{22}$ & $e_{23}$ & $e_{24}$ \\
\hline Parameters & $c_{2} c_{4} c_{3} c_{1}$ & $c_{2} c_{4} c_{3} c_{4}$ & $c_{2} c_{4} c_{3} c_{5}$ & $c_{2} c_{6} c_{3} c_{1}$ & $c_{2} c_{6} c_{3} c_{4}$ & $c_{2} c_{6} c_{3} c_{5}$ \\
\hline$e_{j}$ & $e_{25}$ & $e_{26}$ & $e_{27}$ & $e_{28}$ & $e_{29}$ & $e_{30}$ \\
\hline Parameters & $c_{3} c_{1} c_{3} c_{1}$ & $c_{3} c_{1} c_{3} c_{4}$ & $c_{3} c_{1} c_{3} c_{5}$ & $c_{3} c_{2} c_{3} c_{1}$ & $c_{3} c_{2} c_{3} c_{4}$ & $c_{3} c_{2} c_{3} c_{5}$ \\
\hline$e_{j}$ & $e_{31}$ & $e_{32}$ & $e_{33}$ & $e_{34}$ & $e_{35}$ & $e_{36}$ \\
\hline Parameters & $c_{3} c_{4} c_{3} c_{1}$ & $c_{3} c_{4} c_{3} c_{4}$ & $c_{3} c_{4} c_{3} c_{5}$ & $c_{3} c_{6} c_{3} c_{1}$ & $c_{3} c_{6} c_{3} c_{4}$ & $c_{3} c_{6} c_{3} c_{5}$ \\
\hline
\end{tabular}

The information in $V_{1}, V_{2}, V_{3}$, and $V_{4}$ was then integrated by applying the soft fuzzy set theory, that is, performing "AND" operations on the soft fuzzy sets $\left(F_{1}, C_{1}\right),\left(F_{2}, C_{2}\right)$, $\left(F_{3}, C_{3}\right)$, and $\left(F_{4}, C_{4}\right)$. Then,

$$
\begin{aligned}
(G, E) & =\left(G, C_{1} \times C_{2} \times C_{3} \times C_{4}\right) \\
& =\left(F_{1}, C_{1}\right) \wedge\left(F_{2}, C_{2}\right) \wedge\left(F_{3}, C_{3}\right) \wedge\left(F_{4}, C_{4}\right) .
\end{aligned}
$$

Assuming that the sets $C_{1}, C_{2}, C_{3}$, and $C_{4}$ each provide a parameter to form $E=\left\{e_{1}, e_{2}, \ldots, e_{36}\right\}$, then the parameters of $E$ are as shown in Table 1 .
TABLE 2: Tabular form of soft fuzzy set $(G, E)$.

\begin{tabular}{lcccccccc}
\hline$u_{i j}$ & $e_{1}$ & $e_{2}$ & $e_{3}$ & $e_{4}$ & $e_{5}$ & $e_{6}$ & $e_{7}$ & $e_{8}$ \\
\hline$y x t_{1}$ & 0.4 & 0.4 & 0.4 & 0.4 & 0.4 & 0.4 & 0.4 & 0.4 \\
$y x t_{2}$ & 0.4 & 0.4 & 0.4 & 0.4 & 0.4 & 0.4 & 0.4 & 0.4 \\
\hline$u_{i j}$ & $e_{9}$ & $e_{10}$ & $e_{11}$ & $e_{12}$ & $e_{13}$ & $e_{14}$ & $e_{15}$ & $e_{16}$ \\
\hline$y x t_{1}$ & 0.4 & 0.4 & 0.4 & 0.4 & 0.6 & 0.6 & 0.6 & 0.6 \\
$y x t_{2}$ & 0.4 & 0.4 & 0.4 & 0.4 & 0.5 & 0.5 & 0.5 & 0.5 \\
\hline$u_{i j}$ & $e_{17}$ & $e_{18}$ & $e_{19}$ & $e_{20}$ & $e_{21}$ & $e_{22}$ & $e_{23}$ & $e_{24}$ \\
\hline$y x t_{1}$ & 0.6 & 0.6 & 0.6 & 0.7 & 0.7 & 0.6 & 0.7 & 0.7 \\
$y x t_{2}$ & 0.6 & 0.6 & 0.5 & 0.5 & 0.5 & 0.5 & 0.6 & 0.6 \\
\hline$u_{i j}$ & $e_{25}$ & $e_{26}$ & $e_{27}$ & $e_{28}$ & $e_{29}$ & $e_{30}$ & $e_{31}$ & $e_{32}$ \\
\hline$y x t_{1}$ & 0.6 & 0.6 & 0.6 & 0.6 & 0.6 & 0.6 & 0.6 & 0.6 \\
$y x t_{2}$ & 0.5 & 0.5 & 0.5 & 0.5 & 0.5 & 0.5 & 0.5 & 0.5 \\
\hline$u_{i j}$ & $e_{33}$ & $e_{34}$ & $e_{35}$ & $e_{36}$ & & & & \\
\hline$y x t_{1}$ & 0.6 & 0.6 & 0.6 & 0.6 & & & & \\
$y x t_{2}$ & 0.5 & 0.5 & 0.5 & 0.5 & & & & \\
\hline
\end{tabular}

Since $C_{1} \cap C_{2} \cap C_{3} \cap C_{4}=\varnothing$, the soft fuzzy set $(G, E)$ can be tabulated as shown in Table 2 .

The comparison table CT $=\left(\mathrm{ct}_{x y}\right)_{2 \times 2}$ is computed as shown in Table 3.

The evaluation scores $\operatorname{Score}\left(y x t_{t}\right)$ are computed as shown in Table 4.

As shown in Table 4, Score $\left(y x t_{1}\right)>\operatorname{Score}\left(y x t_{2}\right)$; thus, operation diagram $y x t_{1}$ is superior to diagram $y x t_{2}$. 
TABle 3: Comparison table.

\begin{tabular}{lcc}
\hline $\mathrm{ct}_{x y}$ & $y x t_{1}$ & $y x t_{2}$ \\
\hline$y x t_{1}$ & 36 & 36 \\
$y x t_{2}$ & 14 & 36 \\
\hline
\end{tabular}

TABLE 4: Evaluation scores.

\begin{tabular}{lccc}
\hline & $r_{x}$ & $t_{x}$ & Score $\left(y x t_{t}\right)$ \\
\hline$y x t_{1}$ & 72 & 50 & 22 \\
$y x t_{2}$ & 50 & 72 & -22 \\
\hline
\end{tabular}

\section{Conclusions}

This paper proposed a method for the evaluation of highspeed railway train operation diagrams. The proposed method allows experts to use individual, personalized sets of evaluation indicators, thus allowing overlap among the indicator sets. Excessive and redundant indicators are eliminated via rough set-based attribute reduction, and the remaining important indicators are evaluated. Expert evaluations of train operation diagrams consist of indefinite information in order to more flexibly express subjective judgments. Soft fuzzy set theory is then applied to integrate the information provided by the expert evaluations in order to obtain an overall evaluation. Expert evaluations of some indicators provide uncertain, subjective, or incomplete information (i.e., experts may not evaluate according to all indicators). Therefore, ordinary evaluation methods such as simple weighting, an analytic hierarchy process (AHP), and fuzzy evaluation are not applicable. Soft fuzzy set theory, however, can flexibly solve this problem. The proposed evaluation method is currently applicable only to the evaluation of train operation diagrams that are compiled for the same number of trains, under identical conditions. Plans are underway to adopt relative indicators in the future in order to evaluate train operation diagrams for different numbers of trains or under different conditions. Meanwhile, the proposed method can also be used to evaluate train operation schemes consisting of multiple operation diagrams.

\section{Conflict of Interests}

The authors declare that there is no conflict of interests regarding the publication of this paper.

\section{Acknowledgments}

This research was supported by the National Natural Science Foundation of China (Project nos. 61273242, 61403317, and 61473239), the Fundamental Research Funds for the Central Universities (Project no. 2682015CX043), Science and Technology Plan of China Railway Corporation (Project nos. 2014X004-D, 2015X008-B), Soft Science Foundation of Sichuan province STA of China (Project no. 2015ZR0141), and the Foundation of Sichuan Provincial Education Department (Project no. 14ZB0258).

\section{References}

[1] M. Carey and A. Kwieciński, "Stochastic approximation to the effects of headways on knock-on delays of trains," Transportation Research Part B: Methodological, vol. 28, no. 4, pp. 251-267, 1994.

[2] M. Carey and S. Carville, "Testing schedule performance and reliability for train stations," Journal of the Operational Research Society, vol. 51, no. 6, pp. 666-682, 2000.

[3] I. A. Hansen, "Station capacity and stability of train operations," in Proceedings of the International Conference on Computers in Railways, pp. 809-816, 2000.

[4] R. M. P. Goverde and M. A. Odijk, "Performance evaluation of network timetables using PETER," in Proceedings of the 8th International Conference on Computers in Railways. Computers in Railways, vol. 8, pp. 731-740, Lemnos, Greece, 2002.

[5] R. M. P. Goverde, "Railway timetable stability analysis using max-plus system theory," Transportation Research Part B: Methodological, vol. 41, no. 2, pp. 179-201, 2007.

[6] X. Delorme, X. Gandibleux, and J. Rodriguez, "Stability evaluation of a railway timetable at station level," European Journal of Operational Research, vol. 195, no. 3, pp. 780-790, 2009.

[7] M. Börjesson and J. Eliasson, "On the use of 'average delay' as a measure of train reliability," Transportation Research Part A: Policy and Practice, vol. 45, no. 3, pp. 171-184, 2011.

[8] M. J. C. M. Vromans, R. Dekker, and L. G. Kroon, "Reliability and heterogeneity of railway services," European Journal of Operational Research, vol. 172, no. 2, pp. 647-665, 2006.

[9] R. M. P. Goverde and F. Yan, "Stability and robustness analysis of acyclic timetable," in Proceedings of the 6th International Conference on Railway Operations Modelling and Analysis (RailTokyo '15), Narashimo, Japan, March 2015.

[10] Z. X. Yang and A. Z. Hu, "A study of the dynamic performance \& index system of the train operation diagram," Journal of the China Railway Society, vol. 15, no. 4, pp. 46-56, 1993.

[11] C. H. Tian, "Fuzzy synthetic evaluation on the railway train schedule," Systems Engineering Theory \& Practice, vol. 4, pp. 5054, 1995.

[12] Q. Y. Peng, "A system for evaluation of train diagram elasticity," Journal of Northern Jiaotong University, vol. 33, no. 4, pp. 367371, 1998.

[13] Q. Peng, Y. Zhang, and M. Li, "Methods for evaluation of the equilibrium of train diagrams," Journal of Southwest Jiaotong University, vol. 33, no. 4, pp. 372-377, 1998.

[14] X. Meng and L. Jia, "Train timetable stability evaluation based on analysis of interior and exterior factors information entropy," Applied Mathematics and Information Sciences, vol. 8, no. 3, pp. 1319-1325, 2014.

[15] M. Liu, Researches on Optimization and Evaluation of HighSpeed Railway Timetable, Beijing Jiaotong University, Beijing, China, 2014.

[16] J.-J. Ma, H. Xu, S.-J. Hu, and Z.-X. Chen, "Study of index evaluation system of train working diagram on Jinghu high-speed railway line," Journal of Northern Jiaotong University, vol. 27, no. 5, pp. 46-50, 2003.

[17] T. Zhu, H. Wang, and B. Lu, "Method for optimally selecting the train diagram of passenger dedicated line based on Vague sets," China Railway Science, vol. 32, no. 4, pp. 122-127, 2011.

[18] J. J. Bao, The Evaluation Theory and Method of High-Speed Train Diagram, Southwest Jiaotong University, Chengdu, China, 2012. 
[19] H. Sipilä, "A simulation based framework for evaluatingeffects of infrastructure improvements onscheduled and operational delays," in Proceedings of the 6th International Conference on Railway Operations Modelling and Analysis (RailTokyo), International Association of Railway Operations Research, 2015.

[20] K. Keiji, H. Naohiko, and M. Shigeru, "Simulation analysis of train operation to recover knock-on delay under high-frequency intervals," Case Studies on Transport Policy, vol. 3, no. 1, pp. 92-98, 2015.

[21] A. Higgins and E. Kozan, "Modeling train delays in urban networks," Transportation Science, vol. 32, no. 4, pp. 346-357, 1998.

[22] M. A. Salido, F. Barber, and L. Ingolotti, "Robustness in railway transportation scheduling," in Proceedings of the 7th IEEE World Congress on Intelligent Control and Automation (WCICA '08), pp. 2880-2885, Chongqing, China, June 2008.

[23] Y.-J. Yang and Y.-Q. He, "Evaluation of stability of railway working diagram based on max-plus algebra," Journal of the China Railway Society, vol. 31, no. 4, pp. 14-19, 2009.

[24] L. Y. Meng, Z. X. Yang, H. Y. Li, and J. An, "Analysis on the dynamic performance of PDL train working diagram based on train path layout," China Railway Science, vol. 31, no. 2, pp. 9095, 2010.

[25] Z. Jiang, C.-H. Hsu, D. Zhang, and X. Zou, "Evaluating rail transit timetable using big passengers' data," Journal of Computer and System Sciences, vol. 82, no. 1, part B, pp. 144-155, 2016.

[26] Z. Pawlak, "Rough sets," International Journal of Computer \& Information Sciences, vol. 11, no. 5, pp. 341-356, 1982.

[27] W. X. Zhang, W. Z. Wu, and J. Y. Liang, Rough Set Theory and Method, Science Press, Beijing, China, 2001.

[28] D. Molodtsov, "Soft set theory-first results," Computers and Mathematics with Applications, vol. 37, no. 4-5, pp. 19-31, 1999.

[29] F. Yue, Z.-P. Su, Y. Lu, and G.-F. Zhang, "Comprehensive evaluation of software quality based on fuzzy soft sets," Systems Engineering and Electronics, vol. 35, no. 7, pp. 1460-1466, 2013. 


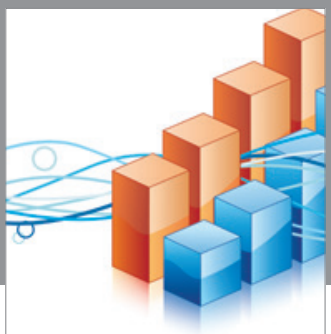

Advances in

Operations Research

vatem alat4

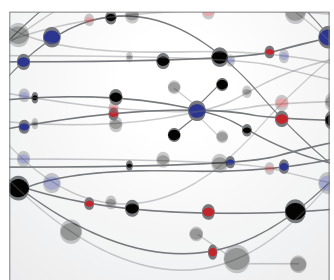

\section{The Scientific} World Journal
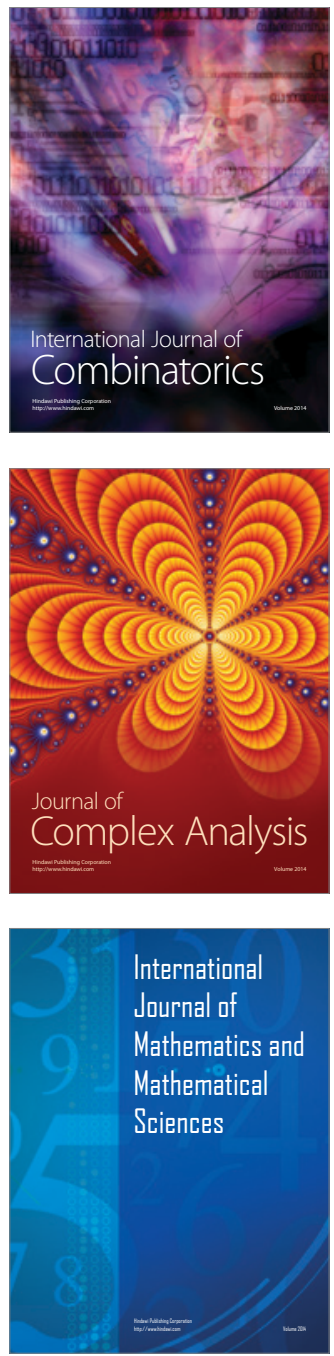
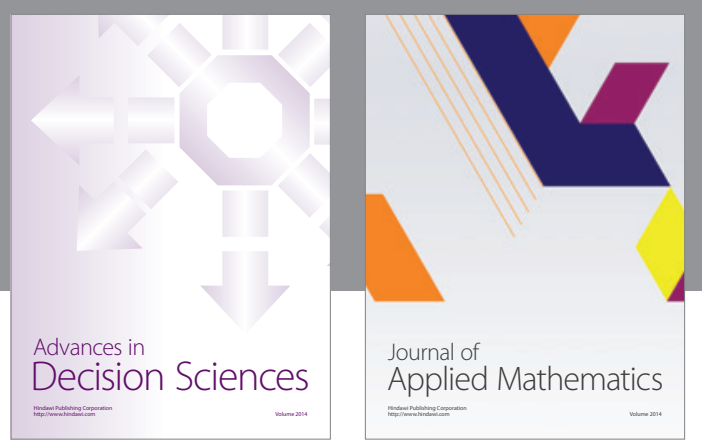

Algebra

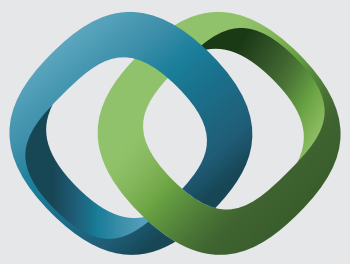

\section{Hindawi}

Submit your manuscripts at

http://www.hindawi.com
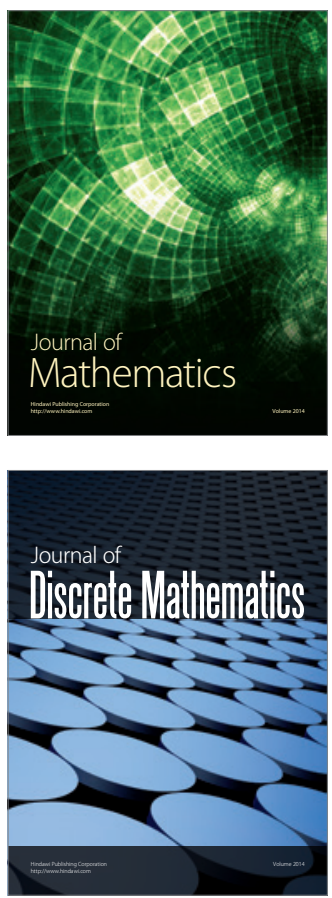

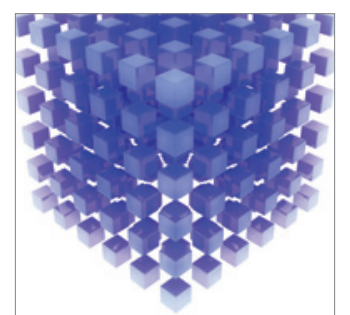

Mathematical Problems in Engineering
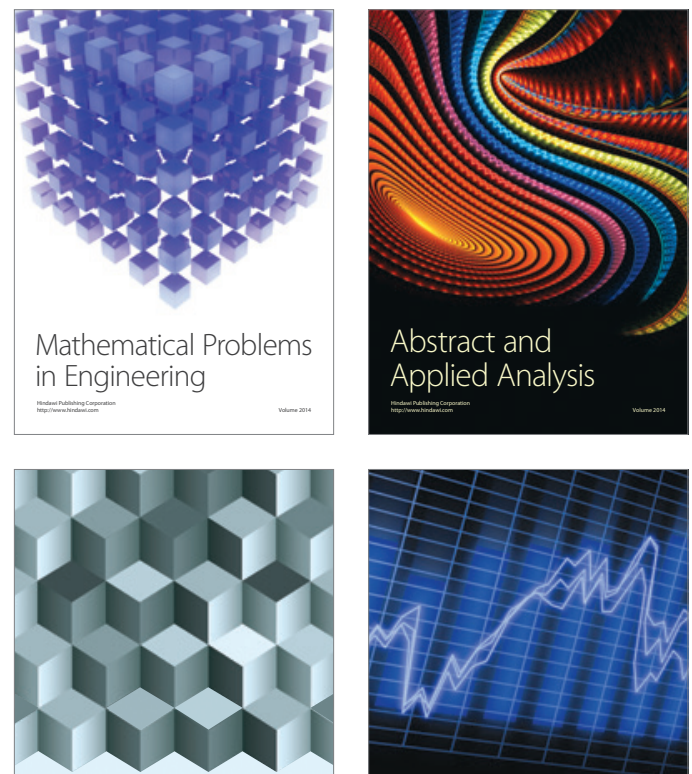

Journal of

Function Spaces

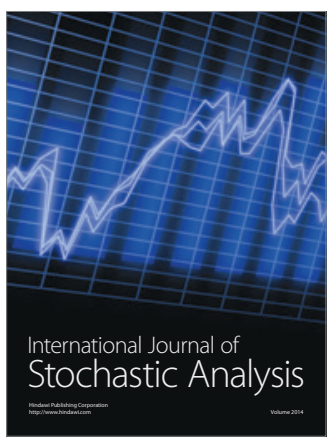

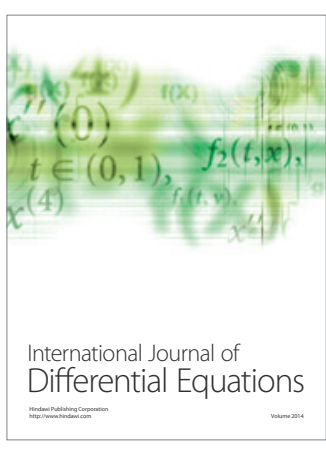
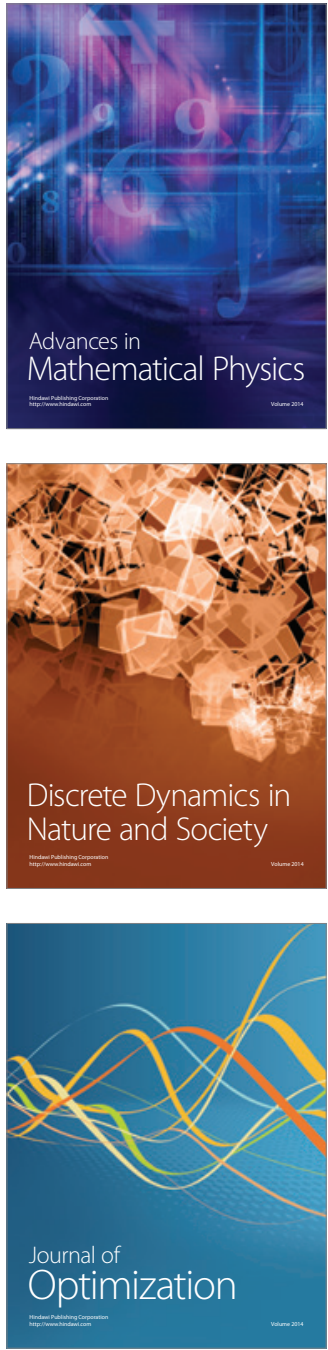